\title{
Troubles dans les frontières: divisions genrées et divisions territoriales chez Jacques Abeille
}

Ivanne Rialland

\section{(2) OpenEdition}

\section{Journals}

Édition électronique

URL : http://journals.openedition.org/studifrancesi/16106

DOI : 10.4000/studifrancesi. 16106

ISSN : 2421-5856

Éditeur

Rosenberg \& Sellier

\section{Édition imprimée}

Date de publication : 1 juillet 2019

Pagination : 87-99

ISSN : 0039-2944

\section{Référence électronique}

Ivanne Rialland, «Troubles dans les frontières: divisions genrées et divisions territoriales chez Jacques Abeille », Studi Francesi [En ligne], 187 (LXIII | I) | 2019, mis en ligne le 01 avril 2020, consulté le 25 janvier 2021. URL : http://journals.openedition.org/studifrancesi/16106 ; DOI : https://doi.org/ 10.4000/studifrancesi. 16106

\section{(c) (i) (9)}

Studi Francesi è distribuita con Licenza Creative Commons Attribuzione - Non commerciale - Non opere derivate 4.0 Internazionale. 


\title{
Troubles dans les frontières: divisions genrées et divisions territoriales chez Jacques Abeille
}

\begin{abstract}
Conceived at first as a "kind of philosophical tale", Jacques Abeille's Les Jardins statuaires (1982) is the beginning of a novel cycle, Le Cycle des contrées, which offers an adventurous and ethnographic journey through an imaginary territory. The ethnographic description conveys a criticism of our Western civilization, based on rationality and technics. Le Cycle des contrées, with its narrators "between two worlds", presents repeated trespassing and merging of the fictive territorial frontiers, and that way enlightens and celebrates the margins of those societies. From that point of view, these books may appear to be promoting queer zones. But this enhancement of margins supposes, indeed, the persistence of frontiers. There is a paradoxical staging of territorial and cultural bonds, that gender relations reveal. Gender relations in theses fictive societies are analysed and criticized, but, in the end, sex difference and heterosexuality are given as biological data. This supposed natural complementarity between sexes is, according to Jacques Abeille's novels, the ultimate basis of alterity, as opposed to the deadly uniformity of Terrèbre civilization. Furthermore, women's alienation itself is positively described, as the way of producing and preserving their singularity. As the domination is always negatively perceived, the margins have to be maintained in their inferior position in order to preserve their positivity.
\end{abstract}

D'abord pensé comme une «manière de conte philosophique» ${ }^{1}$, Les Jardins statuaires de Jacques Abeille (1942 -) forme la première pierre d'un cycle romanesque, Le Cycle des contrées ${ }^{2}$, dont le développement se confond avec l'exploration d'un territoire imaginaire que figure aux yeux du lecteur, à partir de 20083, la carte tracée par Pauline Bergeron, reprise depuis de volume en volume. La place prise par l'observation et la description des mœurs des populations rencontrées par des narrateurs itinérants, voyageur, linguiste, ethnologue ${ }^{4}$, souligne assez que l'intérêt de ces textes réside au moins autant dans la découverte des «contrées» que dans l'intrigue romanesque. Cette ethnographie fictive, cohérente bien qu'éclatée

(1) J. Abeille, in F. Schuiten et J. Abeille, Les Mers perdues, Paris, Attila, 2010, p. 92.

(2) Pour une chronologie du Cycle des contrées et un commentaire de sa structuration, voir E. PERCHOC, Une chronobibliographie et A. Besson, Chronique(s) d'un cycle, in Le Dépossédé (territoires de Jacques Abeille), dir. A. Laimé, Paris, Le Tripode, 2016, respectivement pp. 103-105 et 107-126. Nous parlerons ici essentiellement des Jardins statuaires (1 ${ }^{\text {re }}$ éd. 1982, Paris, Attila, 2010), des Barbares (Paris, Attila, 2011), de La Barbarie (Paris, Attila, 2011), des Voyages du fils (nouv. éd., Paris, Le Tripode, 2016 - c'est cette édition que nous utiliserons donc, sauf mention contraire), de La Grande Danse de la réconciliation (Paris, Le Tripode, 2016) et des Carnets de l'explorateur perdu (Toulouse, Édition Ombres, 1993).

(3) Elle est insérée sur un feuillet à part dans l'édition de 2008 des Voyages du fils (Deleatur/Ginkgo éditeur, 2008), proposant un «signal important d'unité cosmogonique» pour ce cycle, dont l'apparition est «décisive» pour Anne Besson (Chronique(s) d'un cycle cit., p. 117).

(4) Voyageur pour Les Jardins statuaires, linguiste pour Les Barbares et La Barbarie. L'élève de ce linguiste, Ludovic Lindien, apprenti ethnologue, est le narrateur des Voyages du fils, des Carnets de l'explorateur perdu, de La Grande Danse de la réconciliation, et l'auteur du Veilleur du jour (1 ${ }^{\mathrm{re}}$ éd.: Flammarion, 1986), qui raconte l'histoire de son père, Laurent Barthe. 
entre différents volumes et différentes voix, visiblement nourrie par une fréquentation des écrits ethnologiques - l'écriture romanesque en reprenant des thèmes bien reconnaissables: rites funéraires, règles matrimoniales, interdits, rites d'initiation, usage des masques - est structurée par une opposition globale de sociétés traditionnelles - les nomades ou «barbares» (appelés encore les «cavaliers»), les bûcherons, les charbonniers, les bergers, les jardiniers, les hulains - à la ville de Terrèbre, analogon critique de notre civilisation moderne occidentale. Le cycle romanesque déploierait dès lors par le biais de la fiction la critique d'une société technicienne - d'emblée suggérée par la dédicace des Jardins statuaires à Jacques Ellul, l'auteur du Système technicien (1977) - véritable barbarie, au regard de sociétés entretenant un rapport plus authentique à elles-mêmes et leur environnement. Si cette interprétation des romans d'Abeille est juste, elle est néanmoins trop simple face à une œuvre qui fait de la faille, du creux une clé de sa poétique. Mettant en scène des narrateurs «entre deux mondes» ${ }^{5}$, multipliant les franchissement et les brouillages de frontières, Le Cycle des contrées semble à certains égards ne ménager des lignes de fracture que pour mieux déployer un éloge - et une érotique - de la marginalité, qui nécessite pour se déployer le maintien de ces mêmes frontières. Ce rapport paradoxal aux frontières territoriales et culturelles se laisse très nettement saisir à travers la construction et la critique des rapports de sexe dans les différentes sociétés imaginées: le devenir-autre que peuvent performer culturellement les narrateurs, notamment, ne se pense que sur le fond d'une complémentarité fondamentale homme/femme dont l'axiologie est inversée, mais qui est maintenue dans une polarité nécessaire à cette valorisation des marges, qui ne s'oppose pas au conservatisme moral et politique de ce monde romanesque, mais l'implique.

\section{Troubles dans les identités}

Le roman fondateur du Cycle des contrées, Les Jardins statuaires, permet de montrer l'intrication des questions culturelles et de genre tout en mettant en évidence la consistance ethnologique de cet univers fictif. Le territoire des jardins statuaires - où les statues sortent de terre derrière les murs clos des domaines - paraît d'abord une société harmonieuse sous le regard du voyageur, narrateur à la première personne du roman. C'est l'intérêt du voyageur pour la place des femmes qui fait peu à peu apparaître son injustice et sa violence rentrée. Cantonnées avec les enfants à un espace séparé des domaines, d'abord décrit comme un «éden»', elles se révèlent réduites au statut de monnaie d'échange par un système de mariages croisés, les jeunes hommes se devant de prendre femme dans un autre domaine que le leur, afin de perpétuer la force de travail de chaque domaine et les alliances entre ceux-ci:

Le souci principal d'un couple est donc, dès la naissance de leur enfant, de lui assurer le mariage en bon accord avec les autres. Car le garçon est perdu pour le domaine de sa mère; il est donc souhaitable qu'il ait une sœur qui assure la venue d'un élément mâle pour le remplacer. Tout ce commerce implique évidemment des démarches d'un domaine à l'autre. Celles-ci se sont réduites par l'effet du tissage de relations qu'opèrent les déplacements des hommes. Il en résulte, au bout du compte, que le mariage d'un homme est assez tôt déterminé. [...]

(5) C'est ainsi que Félix qualifie le professeur, narrateur des Barbares et de La Barbarie, in Les Voyages du fils cit., p. 152.

(6) J. ABEILle, Les Jardins statuaires cit., p. 124. 
Qui que soient les parents de cette fille, née sur le même domaine que lui, elle est sa sœur. On n'épouse pas sa sœur. Et, même, si on pouvait envisager sans répugnance un tel scandale, que deviendraient alors les alliances?

Bon lévi-straussien, l'informateur du narrateur a conscience que le tabou de l'inceste est déterminé par la volonté de construction sociale ${ }^{8}$. Le sort réservé aux femmes célibataires - exclues des domaines, accueillies dans des «hôtels» où elles sont prostituées - met par ailleurs à mal la symétrie illusoire entre les femmes et les hommes, dont le célibat est certes problématique, mais apparaît comme un bénéfice pour le domaine dont ils sont originaires. Pour reprendre l'expression de LéviStrauss, les femmes sont les «biens du groupe par excellence»': si, dans les jardins statuaires, ce sont les hommes qui circulent, ce sont les femmes qui sont «l'objet de transactions $\gg^{10}$, dont la chair est vendue lorsqu'elle ne peut être échangée - et Gayle Rubin de montrer les implications de l'analyse de Lévi-Strauss en termes de rapport de genres ${ }^{11}$. Il y aurait ainsi quelque chose de profondément queer dans l'entreprise du voyageur, en tant que le queer propose «une théorie de la production de la marginalisation et de la marge, du fait d'être "in" et "out" $»^{12}$, non seulement parce que ses questionnements font apparaitre l'oppression que produit ce fonctionnement social, mais aussi parce qu'ils rendent visibles et compréhensibles des positions dont la marginalité est tue: celle de l'hôtelier dont l'hôtel n'accueille pas de prostituées, celle du guide du voyageur qui fréquente un tel hôtel sans y rechercher de rapports sexuels.

Le voyageur vient de plus perturber activement cette société en soustrayant des femmes au système régissant leur domination. C'est d'abord le cas de Vanina, qui devient sa compagne en dehors de ce système d'alliances, avec qui il s'installe à l'hôtel sans qu'elle ne soit une prostituée. C'est aussi le cas des filles des prostituées, à qui, devenu «gardien du gouffre» ${ }^{13}$, il fabrique en masse les masques protecteurs rituels leur permettant d'échapper au destin de leurs mères. Il élève en outre la nièce du prince barbare, Licia, fille d'une prostituée, en la faisant non seulement échapper à l'espace des hôtels, mais aussi au système des domaines ${ }^{14}$, donnant jour, en somme, à la première femme libre de cette civilisation fictive ${ }^{15}$. Or, c'est ce même rôle de perturbation qu'il occupe au regard de l'opposition entre jardiniers et barbares: il va à la rencontre du chef barbare qui menace les domaines, et vérifie ainsi la légende

(7) Ibid., pp. 73-74.

(8) «[... il fournit le moyen de lier les hommes entre eux, et de superposer, aux liens naturels de la parenté, les liens désormais artificiels, puisque soustraits au hasard des rencontres ou à la promiscuité de l'existence familiale, de l'alliance régie par la règle», C. LÉVI-STRAuss, Les Structures élémentaires de la parenté (1947), Paris-La Haye, Mouton \& Co, 1967, p. 550.

(9) Ibid., p. 549.

(10) G. RuBIn, L'économie politique du sexe: transactions sur les femmes et systèmes de sexe/genre, «Les Cahiers du CEDREF», 7, 1998, mis en ligne le 26 janvier 2010, http://cedref.revues.org/171 (consulté le 20 septembre 2017), $\mathbb{3} 34$.

(11) «[...] ce sont les hommes, qui les donnent et les prennent, qui sont liés entre eux, la femme étant un véhicule de la relation plutôt qu'un partenaire. [...] Et ce sont les partenaires, et non les présents, auxquels l'échange réciproque confère son pouvoir quasi mystique de lien social», ibid.

(12) M.-H. Bourcier, Le Queer Savoir. Epistémologie des espaces de savoir et des disciplines: le point de vue subalterne, in Queer Zones. Politiques des identités sexuelles et des savoirs, 3 e éd., Paris, Éditions Amsterdam, 2011, p. 152.

(13) Le gardien du gouffre est responsable de la destruction des statues malades, précipitées du haut d'une falaise. Dans ce monde de pierre, il est le seul à pratiquer la ferronnerie, et martèle ainsi des masques dont la possession permet aux filles de prostituées d'être accueillies sur un domaine.

(14) «Licia n'appartient à aucun monde», J. ABEILle, Les Barbares cit., p. 436.

(15) Les cavalières mènent certes une vie libre, mais celle-ci semble ne correspondre qu'à un aspect seulement de leur existence: la plupart d'entre elles seraient des femmes des jardins statuaires résidant sur les domaines, voir J. ABEILle, Les Jardins statuaires cit., pp. 235-236. 
selon laquelle il serait un adolescent enfui des domaines. La situation narrative de l'épisode - placé entre les deux visites du voyageur au domaine de Vanina - renforce la similarité de fonction du voyageur, agent d'un brouillage des frontières.

On retrouve cette même intrication dans l'histoire de Laurent Barthe que retracent Les Voyages du fils. Terrébrin envoyé chez les forestiers pour susciter une résistance contre l'invasion barbare, Laurent Barthe tisse des liens entre charbonniers, bûcherons, bergers, d'une part, et d'autre part perturbe là aussi le système de relations des genres: il soustrait une jeune fille au violent rituel de défloration de cette société, les vierges, filles des bûcherons et des femmes du village, étant lancées en groupe dans la forêt pour être pourchassées et violées par les charbonniers - société exclusivement masculine. Or, Laurent ne commet là une transgression que parce qu'à ce moment précis, il occupe la fonction de bûcheron, la transgression du système des genres étant indissociable d'une transgression culturelle. Comme le voyageur, Laurent donne jour ce faisant à un hapax: une fille de bûcheron qui, suggère le texte, aurait été déflorée par un bûcheron et, surtout, dans une passion réciproque ${ }^{16}$.

\section{Une révolution queer avortée}

Ces hommes de l'entre-deux que sont les héros et narrateurs du Cycle des contrées vivent de plus un temps troublé qui semble rendre possible un complet rebrassage des cartes. Le Cycle des contrées organise sa narration autour de l'invasion barbare dont il met en scène les préparatifs, le déroulement et les retombées. Menace pesant sur Les Jardins statuaires et Le Veilleur du jour, cette invasion est en même temps la promesse d'un renouveau, pour la civilisation décadente qu'est évidemment Terrèbre, mais aussi les Jardins statuaires, comme le montre le roman qui leur est consacré. Les germes de ce bouleversement proviennent, d'une façon qui engage profondément le sens de l'œuvre, d'une concentration de l'attention sur les marges, voire les déviances de ce monde imaginaire, que le voyageur rend dicible ${ }^{17}$.

Ce n'est ainsi pas le voyageur qui provoque ce trouble dans les domaines conduisant des jeunes gens à fuir pour rejoindre les barbares, mais il en fait un objet central d'attention et de discours. Par le lien qu'il permet d'établir entre le centre et cette marginalité à la fois spatiale (les marches des domaines) et générationnelle (la jeunesse), il met d'ailleurs fin à ce mouvement centrifuge ${ }^{18}$. De même, ce n'est pas lui qui provoque la sortie de Vanina du système des mariages: c'est elle-même qui refuse le fiancé qui lui était attribué. Cependant, le voyageur l'arrache à cette situation doublement marginale - marginalité de son domaine frontalier, déviance de sa situation matrimoniale - et même taboue, par son contact avec les statues folles de son domaine. Il permet d'être raconté à ce secret honteux, qui pèse sur le roman dès son début puisqu'il détermine de façon tacite les relations entre le guide du voyageur - le fiancé repoussé - et son hôte l'aubergiste - frère de Vanina. Il ramène enfin Vanina au centre

(16) ID., Les Voyages du fils cit., p. 64.

(17) «Comme partout où tu passes, je vois ici des êtres doués de langage et qui désirent parler», souligne Vanina à l'adresse du voyageur (J. ABEILle, Les Jardins statuaires cit., p. 357).

(18) «[...] ce n'était pas que des domaines en perdition que ceux-ci s'étaient enfuis, leurs exodes fracassants navraient l'ensemble du pays d'une hémorragie continuelle tandis que de toute part s'enflait la rumeur qui les appelait vers les steppes. Or, [...] le nombre de ces départs n'avait cessé de décroître depuis que je sillonnais la contrée. [...] on m'espérait comme l'ultime chance de voir à nouveau circuler la parole par-dessus l'abîme qui, insensiblement, s'était creusé entre les générations depuis longtemps déjà isolées dans une ignorance hargneuse», ibid., pp. 202-203. 
du pays, la rendant visible, et il lui donne une dignité ${ }^{19}$, le lustre de leur couple se faisant ferment d'une «espérance ${ }^{20}$ pour les femmes des domaines. Le voyageur opère de la sorte non seulement une mise en visibilité des marges, mais contribue aussi à un renversement axiologique, le déviant - le queer - devenant source potentielle de fécondité, comme le perçoit Vanina sur le domaine des statues folles: «Depuis que tu es là, près de moi, il me semble que quelque chose doit venir de ces étrangetés [...]. Quelque part demeure un germe de fécondité. Le plus vivace» ${ }^{21}$.

Cette transformation de la déviance honteuse en prestige affecte le voyageur lui-même, en une manière de transfiguration initiatique, à l'issue de sa lutte avec les statues malades sur le domaine de Vanina - qui fait immédiatement suite à sa rencontre avec le prince barbare, redoublement non explicité de la «souillure» évoquée par le texte: «Revenant d'une plongée dans l'absolue souillure, je pouvais également apparaître aux yeux de ces hommes très traditionalistes comme un grand maudit ou comme un héros sacré, et c'est dans cette dernière direction que Vanina, par ses bons rapports avec les femmes, fit basculer le jugement commun» ${ }^{22}$.

Si l'action du voyageur est suffisante pour redonner aux Jardins statuaires l'énergie de résister aux barbares, la révolution entrevue des mœurs ne se produit pourtant pas. Devenu gardien du gouffre avant de disparaître, le voyageur accroît sa propre marginalité, en se coulant dans ce rôle par définition solitaire, tandis que Vanina, dans Les Barbares, est une figure certes prestigieuse, mais profondément isolée, vivant à l'écart des femmes comme des hommes des Jardins statuaires ${ }^{23}$. Globalement, dans Le Cycle des contrées, toutes les figures potentiellement révolutionnaires, par leur affranchissement au regard des «bonnes mœurs» - que cela soit du fait de leur connaissance d'autres cultures ou par la liberté de leur érotisme - restent marginales et menacées par le pouvoir grandissant de la civilisation de Terrèbre: le voyageur des Jardins statuaires disparaît, le professeur des Barbares et de La Barbarie est condamné à l'ostracisme, le pornographe Léo Barthe meurt dans la solitude et le dénuement à la fin des Voyages du fils, Laurent Barthe est acculé à la folie. L'invasion des barbares elle-même produit des effets certes positifs, mais temporaires. Les barbares, à bout de souffle, refluent rapidement de Terrèbre, s'absorbent dans les civilisations qu'ils ont un temps bouleversées, tandis que la véritable «barbarie», la civilisation rationnelle et technicienne de Terrèbre, affermit et étend son emprise, balayant les vieilles coutumes. Traversant les Jardins statuaires sur les traces du voyageur, le professeur des Barbares n'y trouve plus de statues, mais de prosaïques légumes ${ }^{24}$ tandis que son retour à Terrèbre le confronte au triomphe d'une atone uniformité:

Plus personne ne s'intéressait aux contrées, on en contestait plutôt l'existence. Tout se passait comme si l'empire de Terrèbre n'avait plus de frontière. Une banalité uniforme s'éten-

(19) Le voyageur répond ainsi à Vanina qui s'inquiète de leur séjour dans l'hôtel: «Il n'est pas question que des conditions difficiles portent atteinte à notre dignité» (ibid., p. 352). Lorsqu'elle insiste, se demandant: «pour qui me prendra-t-on?», il rétorque: «Pour ma compagne, et je te jure bien que nul ne s'y trompera» (ibid.), ce que la suite du texte et du cycle confirme: c'est le statut qui la définit même après la disparition du voyageur. Avec amertume, elle se désigne ainsi dans Les Barbares comme «la veuve du voyageur», J. ABEILLE, Les Barbares cit., p. 344.

(20) «Pendant que je parlais, elle [une prostituée de l'hôtel] nous regardait, Vanina et moi, avec une sorte d'avidité où se lisait de l'espérance», J. ABEILLE, Les Jardins statuaires cit., p. 354.

(21) Ibid., p. 199. Là encore, le voyageur est un révélateur plutôt qu'un initiateur: cette perception de la fécondité des statues monstrueuses est, plus tôt dans le roman, le fait du doyen d'un des domaines qui raconte au voyageur son histoire (ibid., p. $86 \mathrm{~s}$.).

(22) Ibid., p. 345.

(23) ID., Les Barbares cit., p. 332.

(24) Ibid., pp. 310-311. 
dait sur le monde comme une lèpre invisible et tenace et le sentiment de l'altérité humaine s'effaçait au profit d'une intempérante et dérisoire affirmation de soi. Je ne voyais autour de moi nulle invention nouvelle, nul signe de fécondité, seulement une surenchère dans la vanité qui est tout ce qui subsiste quand nulle vraie rencontre n'a plus lieu².

\section{Une altérité essentielle}

Le Cycle des contrées serait dès lors le constat pessimiste de la persistance des identités sclérosantes, hégémoniques, produisant une uniformité culturelle qui donne à cette entreprise romanesque des allures de Tristes tropiques, en nous faisant assister à la disparation progressive des civilisations traditionnelles davantage soucieuses «de l'harmonie de leur site et de la véracité de leur parole» ${ }^{26}$. Il y aurait, ainsi, un bon et un mauvais trouble dans les frontières - le nomadisme des voyageurs s'opposant à l'hégémonie des conquérants - et l'œuvre nous paraît globalement valoriser la persistance d'altérités culturelles tout autant que l'opposition des genres, posée comme une altérité irréductible - résistant donc à toute uniformisation ${ }^{27}$. Le nomadisme culturel des narrateurs ou des héros n'a pas d'équivalent au niveau du genre, alors même que Jacques Abeille est familier des expériences queer de Pierre Molinier (travestissement, auto-sodomie), qu'il interprète comme une tentative de retrouver une indistinction sexuelle archaïque ${ }^{28}$. De façon révélatrice, la folie de Laurent Barthe est provoquée par une simulation de castration, qui le déshumanise sans le déviriliser. Il perd en effet la mémoire, la parole, et vit parmi les cochons, s'accouplant comme une bête avec la veuve qui l'accueille dans sa porcherie ${ }^{29}$. La virilité apparaît comme la composante la plus fondamentale de l'identité, par-delà les cultures, la singularité individuelle, et même l'espèce.

Au mauvais brouillage des frontières auquel procède Terrèbre répond ainsi les jeux de rôles auxquels, dans $\mathrm{La}$ Grande Danse de la réconciliation, se livrent les Hulains, civilisation originelle que Le Cycle des contrées affecte du plus haut degré de valorisation. La Grande Danse de la réconciliation décrit une cérémonie où se mêlent polarisation de genres et polarisation culturelle: elle repose sur la fabrication de quatre masques par un couple hulain, deux fabriqués par la femme, deux par l'homme, l'un des masques jumeaux étant destiné à chaque fois à la vente aux hommes, l'autre étant impliqué dans la cérémonie hulaine. Aux deux niveaux culturel et genré est procédé à un échange: les Hulaines portent lors de la cérémonie les masques fabriqués par

(25) ID., La Barbarie cit., p. 54.

(26) ID., Les Voyages du fils cit., p. 218.

(27) Au-delà du monde fictif des Contrées, cette déploration de l'uniformisation du monde moderne se retrouve dans les textes critiques de Jacques Abeille: «Nous entrions avec cette triste mésaventure [l'édition décevante d'un ouvrage de Pierre Molinier par Pauvert] dans un monde où nous sommes désormais et dont le caractère principal est de ne pouvoir tolérer l'altérité», J. ABEILLE, Pierre Molinier, présence de l'exil, Pessac, Opales - Bordeaux, Pleine Page, 2005, p. 90.

(28) ID., Cherchez la femme (2004), in Pierre Molinier, présence de l'exil cit., 2005, pp. 116-117. L'analyse du plaisir anal comme lieu d'indistinction des sexes dans ce passage - et de cette indécision comme racine de toute vocation artistique - ne se retrouve pas dans Le Cycle des contrées. Au contraire, y est mise en scène l'opposition entre «bonne» et «mauvaise» analité dénoncée par Marie-Hélène Bourcier (Le Queer Savoir cit.): l'étudiant sodomisant la «patronne» et sodomisé dans le même temps par le commissaire sort de l'étreinte humilié - tandis que l'analité concernant la femme de ce trio va de soi: «Alors le commissaire a insulté l'étudiant; il lui a dit qu'il n'était qu'un petit enculé et il lui a rappelé tout ce qu'il avait subi en y prenant plaisir. L'étudiant est devenu blanc - jusqu'au bout il avait essayé de s'imaginer que tout le monde était resté à égalité [...]», J. ABEILlE, Les Chroniques scandaleuses de Terrèbre cit., pp. 89-90.

(29) ID., Les Voyages du fils cit., p. 39 s. 
les Hulains qui sont censés porter à leur envers les rêveries érotiques des hommes ${ }^{30}$, tandis que les masques vendus aux hommes sont habités de «l'innocence orgiaque des enfants [d'Inilo]» ${ }^{31}$. Si la cérémonie performe, doublement donc, un devenirautre, elle relève de façon explicite du jeu, et ne vise qu'à décharger de sa portée conflictuelle une fondamentale opposition des sexes - et des cultures, bien que ce ne soit pas explicite, les masques établissant un lien avec les populations humaines: «Le premier point à ne jamais perdre de vue est la finalité de cette grande danse de la réconciliation: il s'agit d'échapper par un jeu orgiaque à la guerre des sexes qui, toujours menaçante selon les enfants d'Inilo, serait inéluctable si le groupe tout entier ne se livrait de temps à autre à des excès débridés» ${ }^{32}$. La description de ce rite chez les Hulains donne un écho significatif à la déclaration de l'informatrice de Ludovic Lindien, évoquant les liens entre bûcherons et charbonniers: «À l'époque dont nous parlons, négliger leurs différences eût été aussi grave que d'ignorer la répartition de l'humanité en deux sexes. C'eût été une atteinte sacrilège à l'ordre du monde» ${ }^{33}$. Si les échanges sont féconds, et évitent les conflits entre des identités rigidifiées, cette fécondité n'est permise que par le maintien dans leurs différences de ces pôles structurants - néguentropiques - sans lesquelles le monde des contrées tombe dans l'anomie - dans la barbarie terrébrine.

\section{Une dissymétrie naturalisée}

Or, dans ce monde fictif comme dans le nôtre, cette division binaire de l'humanité s'accompagne de relations asymétriques de pouvoir, qui touchent les cultures, mais au premier chef les sexes: leurs rapports hiérarchiques est une donnée universelle de l'anthropologie des contrées - après Lévi-Strauss, c'est Françoise Héritier que l'on rencontre ici. Alors que Jacques Abeille paraît accepter comme un fait de nature la différence des sexes, l'œuvre est plus ambiguë au regard de ces rapports hiérarchiques, objet de discussions et d'interprétations divergentes au sein même de la fiction. On a vu que Les Jardins statuaires mettaient en évidence la marchandisation du corps des femmes impliquée par le système des mariages croisés, et paraissaient les dénoncer à travers le regard du voyageur. En mettant en scène des rituels de fécondité, Les Voyages du fils et Les Barbares interrogent à nouveau l'articulation entre éros et pouvoir qui se joue dans le rapport sexuel, mais d'une manière plus trouble, qui tend à faire conclure, à partir d'une différence sexuelle posée comme biologique, à une asymétrie et une violence inhérentes aux rapports de sexes, qui, du moment qu'elles sont consenties, sont connotées positivement.

Périodiquement, ainsi, les filles nubiles des bûcherons sont envoyées nues dans la forêt pour être pourchassées et violées par les charbonniers. Ce qui symbolise pour la première informatrice de Ludovic Lindien le «centre de noirceur totale» de la communauté des charbonniers et le symbole de «leur vie de bêtes» ${ }^{34}$ est interprété par le

(30) Le masque «est habité des rêves obscènes des hommes que ceux d'avant les hommes imitent en révélant ce qu'ils cachent le plus souvent. D'une certaine manière, le masque capte l'énergie secrète qui hante les adultes des peuples voisins», ibid., p. 24.

(31) Ibid.

(32) Ibid., pp. 23-24.

(33) Ibid., pp. 57-58.

(34) ID., Les Voyages du fils cit., p. 60. 
vieux charbonnier que Lindien interroge ensuite comme un «jeu» $\aleph^{35}$ révélant la «vraie nature ${ }^{36}$ de la femme et de ses rapports à l'homme:

Ce jeu empruntait une apparence de cruauté à la vérité qu'il enseignait à ses participants; il rassemblait en un instant unique et d'autant plus intense d'être sans précédent ni suite tout le drame vital de la conjonction douloureuse et exaltante de l'homme désirant et de la femme désirable. Dans leur dissemblance, comment l'homme aurait-il jamais le beau rôle? Les charbonniers prenaient sur eux le pire. Il s'agissait de connaître l'homme, de connaitre la femme et d'admettre en une fois, aveuglément peut-être, la dissymétrie de leurs relations. ${ }^{37}$

Cette interprétation - qui n'est pas reprise à sa charge par le narrateur, Ludovic Lindien - rapproche ce viol rituel de «l'innocence orgiaque» des hulains, en lui donnant un caractère ludique, et inter-culturel, les charbonniers assumant en somme les fantasmes de la communauté bûcheronne ${ }^{38}$ pour mettre au jour avec «douceur»" dans ce «jeu» la vérité du rapport de sexes. C'est dans un sens similaire qu'est décrypté le viol des cavalières par les cavaliers nomades. La différence des sexes est renforcée là encore par une altérité culturelle, les cavalières provenant pour l'essentiel des jardins statuaires ${ }^{40}$. Cette coutume, décrite au jeune terrébrin Félix par ses compagnons nomades, exige que «quand un cavalier rencontr[e] une de ces femmes sauvages, il l'affront[e] afin de lui imposer une relation charnelle $\gg^{41}$. Ce combat serait souvent une «parodie car la femme [est], dès avant la confrontation, consentante» ${ }^{42}$. Félix est choqué par ce récit, preuve que les nomades qu'il admire ne seraient de fait que des «barbares» ${ }^{43}$, voire que l'humanité tout entière ne recèlerait «aucun bien» ${ }^{44}$. Et c'est cette fois le professeur, soit le narrateur lui-même, qui va justifier cette coutume «d'une injustifiable cruauté» ${ }^{45}$, certes non sans difficulté et embarras, comme un «rite» ${ }^{46}$, renvoyant potentiellement à «un mythe de fécondité» ${ }^{47}$, et révélant in fine l'action de la règle de l'exogamie: «Tout se passait comme si ces femmes fascinantes et mystérieuses se plaisaient à pousser à l'extrême une règle fort répandue qui exigeaient des hommes qu'ils ne s'accouplassent qu'en dehors du groupe restreint auquel ils appartenaient par leur naissance» ${ }^{48}$.

D’une façon révélatrice, Les Carnets de l'explorateur perdu propose une vision inverse, mais parfaitement symétrique de ces rites d'accouplement. Ce recueil de textes, attribué fictivement à Ludovic Lindien, élève du professeur des Barbares, comprend le mémoire d'étude que Lindien a consacré aux cavalières sous sa direction: la cruauté est cette fois celle des cavalières, chasseresses qui tuent leurs partenaires considérés comme du «gibier» ${ }^{49}$. Là aussi, ces appariements paraissent une forme maximaliste

(35) Ibid., pp. 79 et 80.

(36) Ibid., p. 79.

(37) Ibid., p. 80.

(38) Le passage, évoquant «l'innocence des enfants» (ibid.) pour qualifier la mauvaise foi des bûcherons, crée par cette expression antiphrastique un pont supplémentaire avec le rite hulain: les vrais innocents, proches en cela des Hulains, seraient les charbonniers.

(39) Ibid.

(40) Voir ID., Les Barbares cit., p. 390 et Les Jardins statuaires cit., p. 236.

(41) ID., Les Barbares cit., p. 391.

(42) Ibid.

(43) Ibid., p. 393.

(44) Ibid., p. 394.

(45) Ibid.

(46) Ibid.

(47) Ibid., pp. 394-395.

(48) Ibid., p. 395

(49) ID., Les Carnets de l'explorateur perdu cit., p. 38. 
d'exogamie qu'exige la survie d'une «ethnie strictement féminine» ${ }^{50}$ - puisque telle est l'interprétation de Ludovic Lindien, qui diffère donc de la version proposée dans Les Jardins statuaires et Les Barbares où les cavalières ne constituent pas un peuple à proprement parler. Dans les deux cas, qu'elles soient chasseuses ou chassées, les cavalières viennent révéler la «panique» inhérente à l'étreinte amoureuse, en «consonance avec l'élan de la chasse qui trouve dans une telle rencontre son dévoilement» ${ }^{51}$, et qui suppose entre les partenaires «la dissymétrie de leurs relations» ${ }^{52}$.

\section{Une marginalité féconde: la production d'une culture féminine occulte}

En réalité, dans le cas des rencontres avec les cavalières, on ne peut parler d'exogamie: il ne s'agit pas de mariages produisant des échanges entre communautés, mais de rencontres sexuelles éphémères, qui n'affectent pas la composition du groupe. Cette utilisation impropre du terme «exogamie» vient souligner ce redoublement de l'altérité sexuelle par une altérité culturelle $e^{53}$. C'est d'ailleurs en réalité la première qui produit la seconde: alors que les cultures traditionnelles sont vidées de leur substance par Terrèbre, subsiste et résiste une culture féminine, produite par la transformation de la dissymétrie sexuelle en marginalité sociale.

L'hétérogénéité culturelle du couple formé par le déserteur terrébrin et la cavalière dans Les Carnets de l'explorateur perdu est ainsi moins un hapax qu'un révélateur: «Il me parut bientôt que notre couple ne différait des autres, où les partenaires partageaient les mêmes traditions, que du fait d'une étrangeté avouée car, en somme, toutes les femmes sont mystérieuses même si elles savent assez de ruses pour dissimuler le silence où elles demeurent» ${ }^{54}$. Cette généralité («toutes les femmes») correspond certes à une opposition entre une «nature» féminine et une «nature masculine», mais elle est également une polarisation culturelle. Elle renvoie à une «condition féminine» ${ }^{55}$ partagée, qui génère une solidarité entre toutes les femmes, par-delà les cultures. Celle-ci est manifestée par le rôle de protectrices que jouent les cavalières envers les petites filles des jardins statuaires ${ }^{56}$, mais aussi par l'existence, suggérée, d'une langue féminine commune. Le professeur, dans Les Barbares, évoque en effet une «langue féminine secrète» ${ }^{57}$ pratiquée par les femmes des nomades. Or, de son côté, l'amant de la cavalière dont Ludovic Lindien reproduit le récit dans Les Carnets de l'explorateur perdu laisse penser que la langue des cavalières est interdite aux hommes, alors que «certaines paysannes de mon pays prenaient langue assez familièrement avec les guerrières $»^{58}$. Cette langue parlée par les cavalières, si l'on suit la leçon des Jardins statuaires et des Barbares (et donc qu'on les considère non comme une ethnie, mais comme un regroupement de femmes de diverses contrées), pourrait bien être cette «langue féminine secrète» qui les unirait alors. En un sens, cette déduction force le texte, en posant comme une assertion quelque chose qui reste toujours de

(50) Ibid., p. 37

(51) ID., Les Barbares cit., p. 395.

(52) ID., Les Voyages du fils cit., p. 80.

(53) On peut remarquer par ailleurs à cet égard la symétrie entre les cavalières et les charbonniers, deux sociétés non mixtes, dont les membres proviennent d'autres sociétés, et dont les rituels d'accouplement mettent à nu la violence du rapport hétérosexuel.

(54) ID., Les Carnets de l'explorateur perdu cit., pp. 29-30.

(55) ID., Les Barbares cit., p. 197.

(56) Ibid., pp. 429-430.

(57) Ibid., p. 97.

(58) ID., Les Carnets de l'explorateur perdu cit., p. 29. 
l'ordre de la suggestion, de la rumeur, de la légende - les narrateurs, masculins, soulignant leur extériorité par rapport aux coutumes féminines. Elle va toutefois selon nous dans le sens d'une œuvre qui ne cesse de montrer que l'enfermement et la soumission des femmes, qui les cantonnent à un entre-soi, donnent jour à des traditions et des représentations autres que celles des hommes ${ }^{59}$. De livre en livre se constitue un univers féminin relativement unifié, posé comme un envers du monde masculin où circulent les narrateurs. La découverte, dans Les Barbares, du fleuve parcourant les Jardins statuaires en est un emblème:

Donnez-vous une représentation schématique de la contrée des Jardins statuaires. Vous verrez un triangle dont le sommet est au sud et la base au nord, triangle fendu en son milieu par le cours du fleuve qui en trace approximativement la hauteur. Il est évident, si l'on considère que cette hauteur et les deux côtés du triangle sont des régions forestières, que nous avons là des voies de circulation occultes dont le point de convergence se trouve sur les bords du lac où se jette le fleuve, ici même $e^{60}$ et aux alentours. Il s'agit donc pour les cavalières d'un lieu de passage et de séjour privilégié ${ }^{61}$.

Le monde de pierre très masculin que parcourt le voyageur dans Les Jardins statuaires, où les femmes, cantonnées à l'arrière des domaines, forment un groupe invisible et presque indicible, révèle un envers végétal, voie de circulation et de communication pour les cavalières - qui potentiellement, comme Licia, circulent aussi de l'intérieur à l'extérieur des domaines - dont la topographie même évoque un sexe féminin. S'il y a bien là des frontières troublées, brouillées - entre des populations féminines de cultures différentes, entre l'intérieur et l'extérieur des domaines - cet envers féminin ne fait que renforcer la polarisation sexuelle de ce monde fictif en suggérant la coordination et la cohérence d'un univers féminin occulte. De façon révélatrice, par exemple, les rites féminins des Hulains, chez qui pourtant les sexes paraissent politiquement égaux, sont non seulement secrets, mais voilés à destination des hommes hulains de discours volontairement trompeurs, préservant le mystère et la spécificité d'un matrimoine ${ }^{62}$.

(59) Voir I. RialLand, Le Corps perdu:penser l'art à l'ère de la barbarie. "Les Barbares" et "La Barbarie" de Jacques Abeille, in Le Dépossédé cit., p. 150. Voir notamment J. ABEILLE, Les Barbares cit., p. 96 s. Philippe Descola, dans La Composition des mondes, formule - sans autre précision - une remarque confortant l'idée que l'ethnographie imaginaire des Contrées est précisément informée: «On a parfois émis l'idée qu'il y a dans les sociétés traditionnelles une opposition entre l'univers masculin et l'univers féminin, sur lequel pourrait reposer une dissociation des représentations du monde», P. DEsCOLA, La Composition des mondes, Paris, Flammarion, 2014, pp. 286-287.

(60) Cet «ici» désigne la maison close où officie l'énonciatrice, Trudi, la mère de Licia, parmi des prostituées dont le statut oscille dans le texte entre sujétion réelle et feinte, dans une ambiguitté similaire à celle des rites évoqués plus haut. Cette ambiguité nous paraît perçue par l'auteur lui-même, qui met en place à plusieurs reprises dans l'œuvre des discussions à propos de la portée misogyne ou féministe de rites, mœurs, récits à caractère érotique. Voir par exemple les discussions autour de l'«Histoire d'Éponine Délimène» (incluse dans L. BARTHE [J. ABEILLE], Les Chroniques scandaleuses de Terrèbre (2008), Paris, Le Tripode, 2016, pp. 101-126) dans Les Voyages du fils cit., p. 228 s.

(61) J. ABEILLE, Les Barbares cit., p. 428.

(62) «Entre cette informatrice et moi s'était instaurée une confiance si complète qu'elle m'a même dispensé quelques lumières sur la cérémonie secrète des femmes, le rite de Bonda que j'ai évoqué naguère. Contrairement à ce que je m'étais figuré d'abord, cette cérémonie est assez peu scabreuse. Certes, on laisse les hommes se représenter les débordements les plus invraisemblables», J. ABEILLE, La Grande Danse de la réconciliation cit., p. 26. Ludovic Lindien évoque ce culte Bonda dans Les Carnets de l'explorateur perdu, en mettant l'accent sur leur mystère, et la curiosité qu'il suscite chez les hommes. Voir J. ABEILLE, Bonda la lune. Cosmogonie et littérature chez les minorités désertiques, in Les Carnets de l'explorateur perdu cit., p. 85. Tout ce texte travaille les catégories du féminin et du masculin. 


\section{Un conservatisme des marges}

Traversant l'ensemble des cultures des contrées, on est ainsi face à une double partition genrée: l'opposition biologique et dissymétrique d'une nature féminine et d'une nature masculine que révèle le rapport des sexes, et l'opposition entre des cultures masculines et une culture féminine, produite dans sa spécificité et sa cohérence par la hiérarchie sociale des genres.

Cette nature féminine est constamment valorisée, dans la mesure où elle représente les forces vivantes de la nature, du corps - il est tout à fait significatif que les cavalières chevauchent nues - contre la rationalité desséchante du masculin ${ }^{63}$. On est là face à un très net féminisme différentialiste, qui, dans ce système hiérarchique des genres, pourrait amener à un renversement des rapports de pouvoir. Les cavalières, en tant que représentantes d'une puissance guerrière féminine pourraient en être l'agent et, comme le suggère le mémoire de Ludovic Lindien évoqué plus haut, généraliser à l'ensemble des femmes le statut de chasseresses qu'elles peuvent occuper vis-à-vis des hommes - et l'on pense alors aux Guérillères de Monique Wittig. De fait, elles se livrent dans Les Barbares à une démonstration de force lors de la scène de la restitution de Licia à sa mère. Face au tenancier de la maison close et à ses sbires, les cavalières s'y déclarent ses protectrices ainsi que celles des petites filles ${ }^{64}$. Cependant, de manière étonnante, elles récusent aussitôt vouloir la guerre: «Vous savez bien qu'il n'est au pouvoir de personne de vouloir la guerre qui règne pérenne en tous lieux. Tout ce qu'un homme digne de ce nom peut vouloir, c'est la paix. Aujourd'hui, nous sommes venues vous offrir la paix ${ }^{65}$. Les cavalières en restent là, et Licia est de la sorte la seule femme «libérée» des contrées.

Il faut donc bien comprendre ce qu'elle déclare, enfant, au voyageur et à Vanina - «Toute femme est faite pour s'échapper» ${ }^{66}$ - et le commentaire qu'en fait cette dernière: «Je sais aujourd'hui qu'elle avait raison et j'ai pu le vérifier ici même, dans le monde clos des femmes. Elles s'échappent sans cesse. Elles s'échappent vers l'intérieur, dans un silence tel qu'on ne sait où elles sont, ou bien elles s'échappent dans la nuit, dans un espace que rien ne borne, en tout cas elles ne coïncident jamais avec le lieu où on veut les contraindre ${ }^{67}$. Cette échappée n'est pas une fuite mettant fin à une situation d'aliénation, elle est un mouvement continué où s'articulent la «nature» de la femme - qui échappe aux hommes - et sa situation d'aliénation où finalement cette nature peut s'épanouir dans toute sa dimension nocturne. La révolte féminine ne semble pouvoir être qu'un mouvement de retrait, de soustraction, essentiellement intérieur. Les filles nouvelles évoquées par Les Carnets de l'explorateur perdu, qu'a fait émerger le côtoiement avec les cavalières, sont des «filles d'apparence maussade», «dont les silences dissimulent mal la révolte intérieure et la fuite hors des normes de la communauté» ${ }^{68}$. Retrait là encore symbolique: seule Licia vit effectivement cette sécession, qui ne fonde pas une société nouvelle, mais est une marginalité poussée à son extrême, puisque, nous l'avons dit, elle «n'appartient à aucun monde», pas même à celui des cavalières.

C'est que la valorisation du féminin est au moins autant portée par sa marginalité que par son association avec les forces créatrices naturelles: ce qui séduit sans cesse

(63) Voir I. Rialland, Le Corps perdu: penser l'art à l'ère de la barbarie cit., pp. 143-157.

(64) J. AbeILle, Les Barbares cit., pp. 429-430.

(65) Ibid., p. 430.

(66) Ibid., p. 339.

(67) Ibid.

(68) ID., Les cavalières, in Les Carnets de l'explorateur perdu cit. p. 34. 
les narrateurs - et généralement les hommes - dans le monde féminin mis en scène est son caractère occulte qui suscite fantasmes et rêveries. Le statut axiologique des cavalières est à rapprocher de celui des cavaliers: ceux-ci, dans Les Jardins statuaires, fascinent dans la mesure où, êtres marginaux dans le monde sédentaire qui est décrit, ils sont les agents potentiels d'une destruction créatrice de l'ordre établi. Dès qu'ils mettent en ouvre cette subversion, qu'ils dominent, leur spécificité se dissout. Terrèbre, en réalité, ne peut que gagner, car elle est moins une civilisation spécifique que le nom donné à la centralité du pouvoir et à ses effets corrupteurs. Les cavalières elles-mêmes, dans la mesure où elles ont accompagné les nomades dans leur conquête du pouvoir, sont menacées par cette provisoire posture de domination: «On voyait à la fin de plus en plus de couples qui eussent ressemblé au notre [sic] si les cavalières étaient restées en position dominante. Mais d'année en année, elles étaient moins aptes à maintenir vivantes leurs terribles traditions et tombaient dans des états de soumission auxquels, en d'autres temps, elles eussent préféré la mort» ${ }^{69}$. C'est à une valorisation globale de la marge que se livre Jacques Abeille, qui ne rend donc pas souhaitable, dans ce monde imaginaire, une libération des femmes, ou une quelconque révolution des dominés: ce qui est out est certes l'objet de toutes les attentions, mais se doit de rester out pour rester désirable - car c'est bien là d'une érotique autant que d'une politique qu'il est question ${ }^{70}$.

\section{Conclusion}

Si quelques personnages ont ainsi le pouvoir, séduisant, de transgresser les frontières et de se mouvoir dans les marges que proposent les Contrées, l'abolition des frontières n'aboutit qu'à une atone uniformité. Plus encore, les rapports de domination que dénoncent à certains égards ces romans sont en réalité essentiels à la mise en scène valorisée, érotisée de la marge: une valorisation de la marge en tant telle implique la persistance non seulement d'une polarisation opposant Terrèbre aux sociétés traditionnelles et le masculin au féminin, mais aussi d'une domination entre ces pôles. Ce faisant, le féminin est placé à la position même de l'art véritable, dont il parait la source, au regard de l'académisme, opposant la même difficulté à une reconnaissance de l'œuvre d'Abeille qu'à une libération politique des femmes. Jacques Abeille lui-même revendique de la sorte la marginalité de son œuvre, se déclarant «plus près du fou littéraire que de toute autre école» ${ }^{71}$, saluant dans ses romans une littérature marginale ${ }^{72}$ à laquelle les volumes du Cycle fictivement s'incorporent ${ }^{73}$, célébrant le «monstre» Molinier ${ }^{74}$, s'identifiant à des écrivains marginaux, et singulièrement à des figures féminines, décrites comme «tout à la périphérie» du mouvement

(69) Ibid., p. 31.

(70) Avec un effet de tourniquet: l'érotisme est marginal politiquement parce qu'érotique - puissance de vie, de désir - mais il est d'autant plus érotique qu'il est marginal - ce qui ne rend pas souhaitable, dans ce monde fictif, un affaiblissement de la censure exercée par Terrèbre. Voir J. AbeILle, Les Voyages du fils cit., pp. 165,182 et 184 .

(71) J. ABEILlE, En France, on condamne l'imagination, propos recueillis par D. Caviglioli, «BiblioObs», 19/11/11, [en ligne: https://bibliobs.nouvelobs.com/romans/20111117.OBS4761/en-france-on-condamne -l-imagination.html] (consulté le 23/11/2017).

(72) Dans Les Voyages du fils est saluée par exemple l'attitude des auteurs du «second rayon» durant l'invasion barbare qui «sauvèrent la langue écrite», ibid., p. 224.

(73) Dans le monde des Contrées, le livre Les Jardins statuaires, notamment, circule ainsi sous le manteau.

(74) Voir J. Abeille, Pour Molinier, le monstre (1966), in Pierre Molinier, présence de l'exil cit., 2005, pp. 13-28. 
surréaliste: «Si je devais m’assigner une place dans le contexte du surréalisme, finalement, aujourd'hui, c'est dans cette nébuleuse - parmi les femmes - que je me situerais $[\ldots] \gg^{75}$. Il s'agit par là de se placer dans une situation de radicale altérité, queer donc, en effet, mais de façon non subversive, essentialiste, dans une volonté de préserver cette étrangeté au monde que toute incorporation à la norme dominante risquerait de détruire ${ }^{76}$. 